future revisions to the script. A substantial discount is available for additional sets. Enquiries should be addressed to "The Director" of the Observatory at the author's address given above.

\title{
References
}

Aller, L.H. 1949, Astrophys. J., 109, 244.

Aller, L.H. 1963, Astrophysics: The Atmospheres of the Sun and Stars, Second Ed.

(Ronald Press: New York), 124.

Aller, L.H. and Jugaku, J. 1956, Astrophys. J., 127, 244.

Dworetsky, M.M. 1980, Q. J. Roy. Astr. Soc., 21, 50.

Roy, A.E. and Clarke, D. 1982, Astronomy: Structure of the Universe, Second Ed. (Adam Hilger: Bristol), ch. 7.

Zeilik, M. and Smith, E.v.P. 1987, Introductory Astronomy and Astrophysics, Second Ed. (Saunders: Philadelphia), ch. 13.

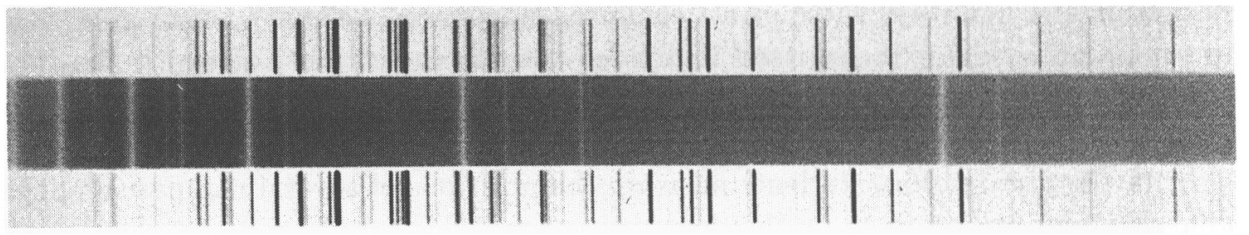

Fig. 1. The spectrogram of the B3V star $\iota$ Herculis used in this experiment (original dispersion $140 \AA / \mathrm{mm}$ ). University of London Observatory photograph.

\section{TELESCOPES FOR STUDENTS}

\section{Roy L. Bishop}

Department of Physics, Acadia University, Wolfville, Nova Scotia, B0P 1X0, Canada

An astronomy class typically is composed of twenty or more students, one instructor, and one telescope. For observing sessions, these numbers mean that at any one moment most of the students are standing, slowly freezing in the dark, waiting their turn at the eyepiece. Moreover, when they do have their 20-second peek, moist breath, a bump by the last observer, or unfamiliarity with the instrument often mean that the object will look hazy, be out of focus, or even be out of view. A couple of sessions under these circumstances can dampen the interest even of keen students. Also, the instructor may be reluctant to let individual students have unsupervised access to the one, expensive, fragile telescope.

Ideally each student would have a telescope during supervised observing sessions. Each telescope should be easy-to-use, rugged, equipped with setting circles, 
and mechanically and optically designed so as to minimize conceptual barriers between its operation and the geometry of the sky. As a step toward this goal, for the introductory astronomy course at Acadia University we have designed and constructed a set of six telescopes (Bishop, 1986).

Each telescope is a modest, wide-field, equatorially-mounted refractor. The objective is an $\mathrm{f} / 5$ achromat with a clear aperture of $74 \mathrm{~mm}$ and is combined with a $16 \mathrm{~mm}$ eyepiece having an apparent field of $69^{\circ}$. This gives a magnification of $23 \times$ and an actual field of $3^{\circ}$. Thus a finder telescope is not required and the field is large enough (and the aperture sufficient) to provide striking views of several objects that cannot be encompassed by the narrower fields of larger telescopes (for example: the Pleiades, the Praesepe cluster, the North America Nebula, the entire sword of Orion with the Great Nebula, the Andromeda Galaxy together with its two companion galaxies, etc).

With these telescopes, angles as small as 10 seconds of arc can be resolved, yet atmospheric turbulence is not a problem. The disk of Jupiter, the rings of Saturn, the double star Mizar, and the Trapezium in the Orion Nebula can be seen. Thus, in addition to providing interesting wide-angle views, the telescopes provide an an introduction to sights that are displayed to better advantage in larger instruments. Even for some objects that are generally considered to require larger instruments (for example, the Triangulum galaxy, and the Crab Nebula), these telescopes provide pleasing views.

The usual neck-saving, $90^{\circ}$ bend in the optical path is provided by a roof (Amici) prism. The two reflections ensure that the image is both upright and correct left-for-right, and thus has the same orientation as the naked-eye view of the sky when students raise their heads. Also, the telescopic view may be compared directly to star charts. This eliminates a source of confusion for students, and, even for experienced observers, is a convenience that must be experienced to be fully appreciated.

Each telescope is mounted on a substantial, portable, three-legged pier and is designed to be used while the observer is comfortably seated on a low stool. There are no clamps or slow-motion controls to bother with: all motions are smooth and restrained by friction in Teflon-lined adjustable bearings. The right ascension and declination dials are large and easy to read, and, to minimize conceptual difficulties, are oriented so that their pointers are parallel to the optic axis of the telescope. Thus, the calibration marks on both dials, extended radially, coincide with the imaginary grid of right ascension and declination on the sky.

Aside from providing more time at the eyepiece for students, these telescopes provide experience that is missing at observing sessions involving one, larger, motordriven telescope that necessarily has been pre-aligned on objects of interest. When they sit down beside one of these basic instruments, students are literally cast adrift among the stars: they have to study the star patterns, think about coordinates, and allow for the turning of the planet beneath their feet. This experience will probably stay with them long after other details learned in class have been forgotten. 

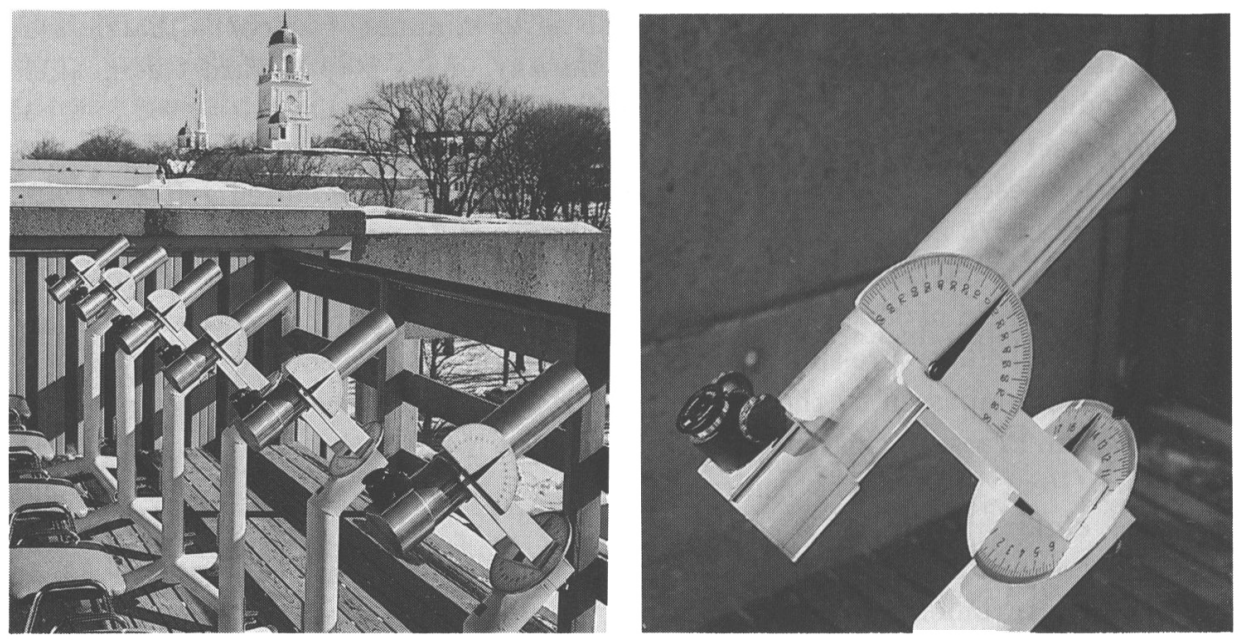

Fig. 1. Telescopes for students. Left: six telescopes mounted on the observing deck. Right: close-up view of one telescope.

\section{References}

Bishop, R.L. 1986, J. Roy. Astron. Soc. Can., 80, No. 4, 211-215.

\section{USING CCD'S IN INTRODUCTORY-LEVEL COLLEGE ASTRONOMY LABORATORIES}

Thomas J. Balonek

Colgate University, Dept. of Physics and Astronomy, Hamilton, New York 13346, U.S.A.

\section{The CCD and Interactive Image Display Systems}

Our university recently purchased a liquid-nitrogen-cooled CCD camera system (from Photometrics Ltd., Tucson, Arizona) which has been installed on our campus' 40-cm multiple access (Cassegrain/Newtonian) telescope. Images are reduced online at the observatory using Photometrics' microcomputer-based analysis software package, which includes operations for standard data acquisition and initial stages of data reduction - including corrections for bias, dark current, and flat fielding. Images are displayed on a 256-level-gray-scale black and white monitor. Additional post-processing can be done either on the CCD system's computer at the observatory 